\title{
Multiomic analysis and immunoprofiling reveal distinct subtypes of human angiosarcoma
}

\begin{abstract}
Jason Yongsheng Chan, ${ }^{1,2,3}$ Jing Quan Lim, ${ }^{4}$ Joe Yeong, ${ }^{5,6}$ Vinod Ravi, ${ }^{7}$ Peiyong Guan, ${ }^{8}$ Arnoud Boot, ${ }^{8,9}$ Timothy Kwang Yong Tay, Sathiyamoorthy Selvarajan, ${ }^{5}$ Nur Diyana Md Nasir, ${ }^{5}$ Jie Hua Loh, ${ }^{5}$ Choon Kiat Ong, ${ }^{4,10}$ Dachuan Huang, ${ }^{4}$ Jing Tan, ${ }^{11}$ Zhimei Li, ${ }^{11}$ Cedric Chuan-Young Ng, ${ }^{11}$ Thuan Tong Tan, ${ }^{12}$ Mikio Masuzawa, ${ }^{13}$ Ken Wing-Kin Sung, ${ }^{14,15}$ Mohamad Farid, ${ }^{1,3}$

Richard Hong Hui Quek, ${ }^{1}$ Ngian Chye Tan, ${ }^{16,17}$ Melissa Ching Ching Teo, ${ }^{16}$ Steven George Rozen, ${ }^{8,9,10}$

Patrick Tan, ${ }^{2,6,10,14}$ Andrew Futreal, ${ }^{18}$ Bin Tean Teh, ${ }^{2,6,10,11,19}$ and Khee Chee Soo ${ }^{16,17}$

'Division of Medical Oncology, National Cancer Centre Singapore, Singapore. ${ }^{2}$ Cancer Science Institute of Singapore, National University of Singapore, Singapore. ${ }^{3}$ SingHealth Duke-NUS Blood Cancer Centre, Singapore. ${ }^{4}$ Lymphoma Genomic Translational Research Laboratory, Division of Cellular and Molecular Research, National Cancer Centre Singapore, Singapore. ${ }^{5}$ Department of Anatomical Pathology, Singapore General Hospital, Singapore. ${ }^{6}$ Institute of Molecular and Cell Biology, Singapore. ${ }^{7}$ Department of Sarcoma Medical Oncology, University of Texas MD Anderson Cancer Center, Houston, Texas, USA. ${ }^{8}$ Integrated Biostatistics and Bioinformatics Programme, ${ }^{9}$ Centre for Computational Biology, and ${ }^{10}$ Program in Cancer and Stem Cell Biology, Duke-NUS Medical School, Singapore. "Laboratory of Cancer Epigenome, Division of Medical Sciences National Cancer Centre Singapore, Singapore. ${ }^{12}$ Department of Infectious Diseases, Singapore General Hospital, Singapore. ${ }^{13}$ Department of Regulation Biochemistry, School of Allied Health Sciences, Kitasato University, Minato City, Tokyo, Japan. ${ }^{14}$ Cenome Institute of Singapore, A*STAR, Singapore. ${ }^{15}$ School of Computing, National University of Singapore, Singapore. ${ }^{16}$ Division of Surgical Oncology, National Cancer Centre Singapore, Singapore. ${ }^{17}$ SingHealth Duke-NUS Head and Neck Centre, Singapore. ${ }^{18}$ Department of Genomic Medicine, University of Texas MD Anderson Cancer Center, Houston, Texas, USA. ${ }^{19}$ Division of Cellular and Molecular Research, National Cancer Centre Singapore.
\end{abstract}

Angiosarcomas are rare, clinically aggressive tumors with limited treatment options and a dismal prognosis. We analyzed angiosarcomas from 68 patients, integrating information from multiomic sequencing, NanoString immuno-oncology profiling, and multiplex immunohistochemistry and immunofluorescence for tumor-infiltrating immune cells. Through whole-genome sequencing ( $n=18$ ), 50\% of the cutaneous head and neck angiosarcomas exhibited higher tumor mutation burden (TMB) and UV mutational signatures; others were mutationally quiet and non-UV driven. NanoString profiling revealed 3 distinct patient clusters represented by lack (clusters 1 and 2) or enrichment (cluster 3 ) of immune-related signaling and immune cells. Neutrophils (CD15+), macrophages (CD68 ${ }^{+}$), cytotoxic T cells (CD8+), Tregs (FOXP3 ${ }^{+}$), and PD-L1+ cells were enriched in cluster 3 relative to clusters 2 and 1. Likewise, tumor inflammation signature (TIS) scores were highest in cluster 3 (7.54 vs. 6.71 vs. 5.75 , respectively; $P<0.0001)$. Head and neck angiosarcomas were predominant in clusters 1 and 3 , providing the rationale for checkpoint immunotherapy, especially in the latter subgroup with both high TMB and TIS scores. Cluster 2 was enriched for secondary angiosarcomas and exhibited higher expression of DNMT1, BRD3/4, MYC, HRAS, and PDCFRB, in keeping with the upregulation of epigenetic and oncogenic signaling pathways amenable to targeted therapies. Molecular and immunological dissection of angiosarcomas may provide insights into opportunities for precision medicine.

\section{Introduction}

Angiosarcomas represent a rare group of soft-tissue sarcomas (1, 2) and are aggressive endothelial cell tumors of vascular or lymphatic origin. These tumors demonstrate remarkable heterogeneity in terms of clinical presentation and behavior and can develop in various anatomical structures, including cutaneous regions of the head and neck, breast, viscera, and bone. Despite their rarity, several well-defined risk factors have been identified. Angiosarcomas arise in distinct clinical settings, either de novo (primary) or following radiotherapy or chronic lymphedema (secondary) (2). Regardless of etiology or anatomical origin, angiosarcomas are

Authorship note: JYC, JQL, and JY are co-first authors and contributed equally to this work.

Conflict of interest: The authors have declared that no conflict of interest exists. Copyright: () 2020, American Society for Clinical Investigation.

Submitted: April 13, 2020; Accepted: July 29, 2020; Published: October 5, 2020.

Reference information: J Clin Invest. 2020;130(11):5833-5846.

https://doi.org/10.1172/JCl139080. characterized by a challenging clinical course with limited treatment options and a dismal prognosis. Contemporary treatment of localized angiosarcoma involves a multimodality approach incorporating surgical resection with wide margins when feasible, as well as radiotherapy and/or chemotherapy in select clinical scenarios. In the metastatic setting, chemotherapeutic agents including paclitaxel, doxorubicin, or targeted agents are typically administered, albeit with limited efficacy or clinical benefit $(2,3)$.

At the molecular level, specific genetic alterations have been correlated with clinical phenotypes, of which MYC amplification in secondary angiosarcoma of the breast is among the best characterized (4). Mutations involving the angiogenesis-related genes PTPRB and/or PLCG1 (5), as well as FLT4 amplification have been observed $(6,7)$. Additionally, radiation-induced and post-lymphedema angiosarcomas were shown to be transcriptomically distinct from primary angiosarcomas in 1 study (8). Between UVassociated and radiation-associated cases, unique genome-wide DNA methylation clusters have been recognized (9). More recently, a patient-partnered research initiative (Angiosarcoma Project 
[ASCproject]) reported high tumor mutation burden (TMB) and dominant UV damage mutational signatures in a subset of patients with angiosarcoma arising from the head and neck (10). This finding suggests that UV-induced mutagenesis may be a causative factor and supports a therapeutic rationale for checkpoint immunotherapy. The importance of checkpoint immunotherapy is further supported by reports of promising responses seen in 2 patients with chemorefractory angiosarcomas with high TMB and dominant UV mutational signatures, generating interest in better characterizing these tumors as well as their immune microenvironment (10).

Therefore, by integrating next-generation multiomic sequencing, NanoString gene expression platforms for immuno-oncology profiling, and multiplex IHC, the current study aimed to investigate different clinical subtypes of angiosarcomas in detail by simultaneous examination of their genomic, transcriptomic, and immune landscapes.

\section{Results}

UV mutational signatures define a unique subset of human angiosarcoma. We performed whole-genome sequencing of 18 angiosarcomas from the head and neck region $(n=13)$, liver $(n=2)$, breast $(n=$ $1)$, abdominal wall $(n=1)$, and thigh $(n=1)$. We observed recurrent mutations in known angiosarcoma-associated genes including TP53 (22\%), KDR (17\%), POT1 (17\%), and PTPRB (17\%) (Figure $1 \mathrm{~A}$ and Supplemental Table 1; supplemental material available online with this article; https://doi.org/10.1172/JCI139080DS1). The median TMB was 1.95 mutations per coding megabase (mt/ $\mathrm{Mb}$ ) (range, 0.06-7.16) and was notably highest in a subset of cutaneous angiosarcomas of the scalp and face (patients AS-13 to AS-18; Figure 1B). A subgroup of angiosarcomas with high TMB was also identified in the ASCproject (Supplemental Figure 1 and ref. 10). Analysis of de novo mutational signatures showed that this subgroup of angiosarcomas harbored a distinctive UV signature (cosine similarity of 0.95 to Catalogue of Somatic Mutations in Cancer [COSMIC] Mutational Signature 7a) (Figure 1C). The other cases (AS-01 to AS-12), including 7 patients with scalp angiosarcoma, had a significantly lower mutation burden (median, 5.04 vs. $0.91, P=0.0007)$. In concordance with this result, the subgroup of tumors with UV mutation signatures (AS-13 to AS-18) exhibited higher levels of $\mathrm{C}>\mathrm{T}$ mononucleotide substitutions and CC>TT dinucleotide substitutions, as well as positive staining for cyclobutane pyrimidine dimers (CPDs) on IHC (Figure 1, D and E, and Supplemental Figures 2-4). In the overall cohort, we observed evidence of UV exposures by positive CPD detection in 17 of 35 (48.6\%) angiosarcomas arising from the head and neck (Supplemental Table 2), and 2 of 5 (40.0\%) cutaneous angiosarcomas arising from the trunk and limbs. None of the visceral or breast angiosarcomas was positive for CPDs (Figure 1F). Among patients with resected head and neck angiosarcomas, those with UV signatures had improved survival outcomes (HR 0.30, 95\% CI, 0.10-0.96, log-rank $P=0.0433$ ) (Figure $1 G$ ).

Analysis of somatic copy number alterations in the same samples (AS-01 to AS-18) identified 37 gained genomic and 68 lost regions (Supplemental Figure 5, A and B). Gene-level copy number analysis showed that patient AS-06, with abdominal wall angiosarcoma following prior irradiation for cervical cancer, harbored amplification of MYC ( $\log _{2}$ fold-change tumor vs. normal,
4.93), though the significance of this finding outside the context of secondary angiosarcomas of the breast remains to be validated (46). Recurrent structural rearrangements observed included those in the chromosome 6 region harboring the HLA complex and chromosome 17 region harboring MAP2K3 (Supplemental Figure $5, C$ and D). Interestingly, patient AS-12 was diagnosed with stage 3 invasive ductal carcinoma of the breast at age 35 , followed by angiosarcoma of the thigh muscle 2 years later. The strong family history suggestive of Li-Fraumeni syndrome was confirmed by a pathogenic germline TP53 (NM_000546) exon 4 c.374C>A heterozygous variant. The mutational spectrum also highly resembles COSMIC Mutational Signature 38 (cosine similarity =0.96) (Supplemental Figure 6).

Distinct clusters of angiosarcoma provide opportunities for precision therapy. We applied the NanoString PanCancer Immuno-Oncology IO360 panel to interrogate gene expression profiles in an expanded cohort of patients and identified 3 distinct clusters characterized by different clinical features, pathway scores, and immune profiles (Figure 2A). The most striking differences across the clusters lay in immune-related pathways. Clusters 1 and 2 were characterized by a relative lack of immune-related signaling and immune cells, as compared with cluster 3 . The tumor inflammation signature (TIS) scores were generally higher compared with TIS scores for most other tumor types reported previously (11), with a median of 6.76 (range, 5.23-9.65) (Figure 2B). TIS scores were highest in cluster 3 compared with those for clusters 2 and 1 (7.54 vs. 6.71 vs. 5.75, respectively; $P<0.0001$ ) (Figure $2 \mathrm{C}$ ).

In terms of similarities across clusters, we noted that primary head and neck angiosarcomas were dominant in clusters 1 and $3(P=0.0138)$, frequently with evidence of UV-related DNA damage $(P=0.0196)$ (Figure $2, \mathrm{D}$ and $\mathrm{E})$. These 2 distinct subgroups remained evident after limiting the analysis to include only angiosarcomas of the head and neck. We identified 2 distinct clusters on the basis of immune-related signals that could be categorized into immune "cold" or "hot" subgroups. The original cluster 1 cases and cluster 3 cases were expectedly categorized as immune "cold" and "hot," respectively, whereas cluster 2 cases were redistributed into immune "cold" (AS-52, AS-03) or "hot" (AS-57, AS-10, AS-44) groups (Supplemental Figure 7A). This classification may provide the rationale for checkpoint immunotherapy especially in the subgroup with both high TMB and TIS scores (Supplemental Figure 7B).

On the other hand, patients in cluster 2 had enrichment of genes in secondary angiosarcomas (6 of 14) with prior radiation exposure $(n=4)$, chronic lymphedema (AS-37), or arising from a thrombosed arteriovenous fistula (AS-48). Five head and neck angiosarcomas were within cluster 2 , of which only 1 was UV related. Notably, cluster 2 angiosarcomas exhibited significant expression levels of DNMT1, MYC, and HRAS $(P<0.01)$ as well as $B R D 3 / 4$ and $P D G F R B(P<0.05)$, in keeping with upregulation of epigenetic and oncogenic signaling pathways that could be further investigated as potential therapeutic targets (Figure $2 \mathrm{~F}$ and Supplemental Tables 3 and 4).

Transcriptomic profiling identifies actionable pathways in angiosarcoma. To survey the transcriptomic landscape further on a global level, we performed RNA-Seq on selected samples and angiosarcoma cell lines, along with matched normal tissue where 
A Nonsynonymous mutation count



B



D

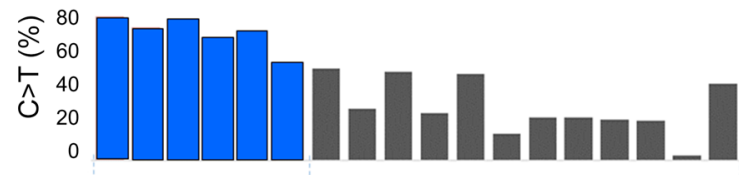

100
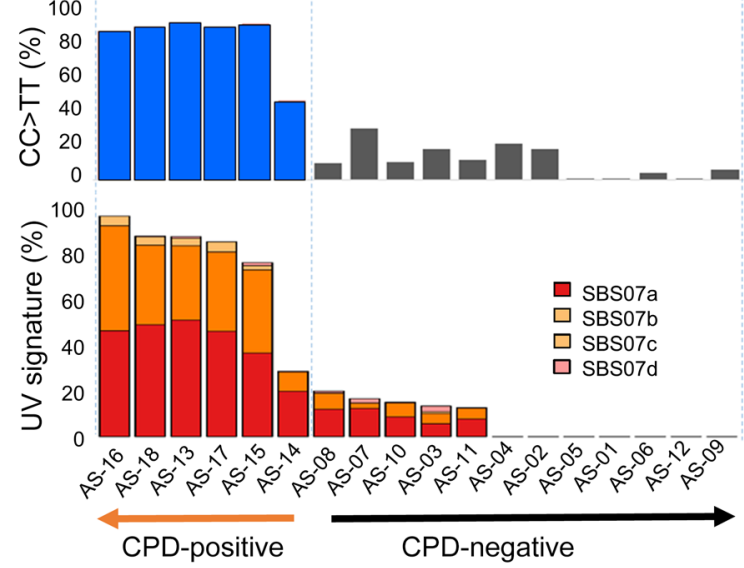

E



$\mathbf{F}$



G



Figure 1. Somatic mutational landscape of human angiosarcoma. (A) Oncoplot of somatic nonsynonymous variants of interest, including recurrent mutations in the known angiosarcoma-associated genes TP53, KDR, POT1, and PTPRB. (B) TMB was highest in a subset of head and neck angiosarcomas (AS-13 to AS-18), which harbored (C-E) distinctive UV signatures (COSMIC Mutational Signature 7a, cosine $=0.95$ ) along with higher levels of C>T mononucleotide substitutions and CC>TT dinucleotide substitutions, as well as positive staining for CPDs on IHC (scale bar: $20 \mu \mathrm{m})$. (F) In the overall cohort, positive CPDs were observed in 17 of 35 (48.6\%) angiosarcomas arising from the head and neck, and 2 of $5(40.0 \%)$ cutaneous angiosarcomas arising from the trunk and limbs. None of the visceral or breast angiosarcomas was positive for CPDs (Fisher's exact, $P<0.0001)$. (G) Among patients with resected head and neck angiosarcomas, those with UV signatures had improved survival outcomes (HR $0.30,95 \% \mathrm{Cl}, 0.10-0.96$, log-rank test, $P=0.0433$ ). 
A

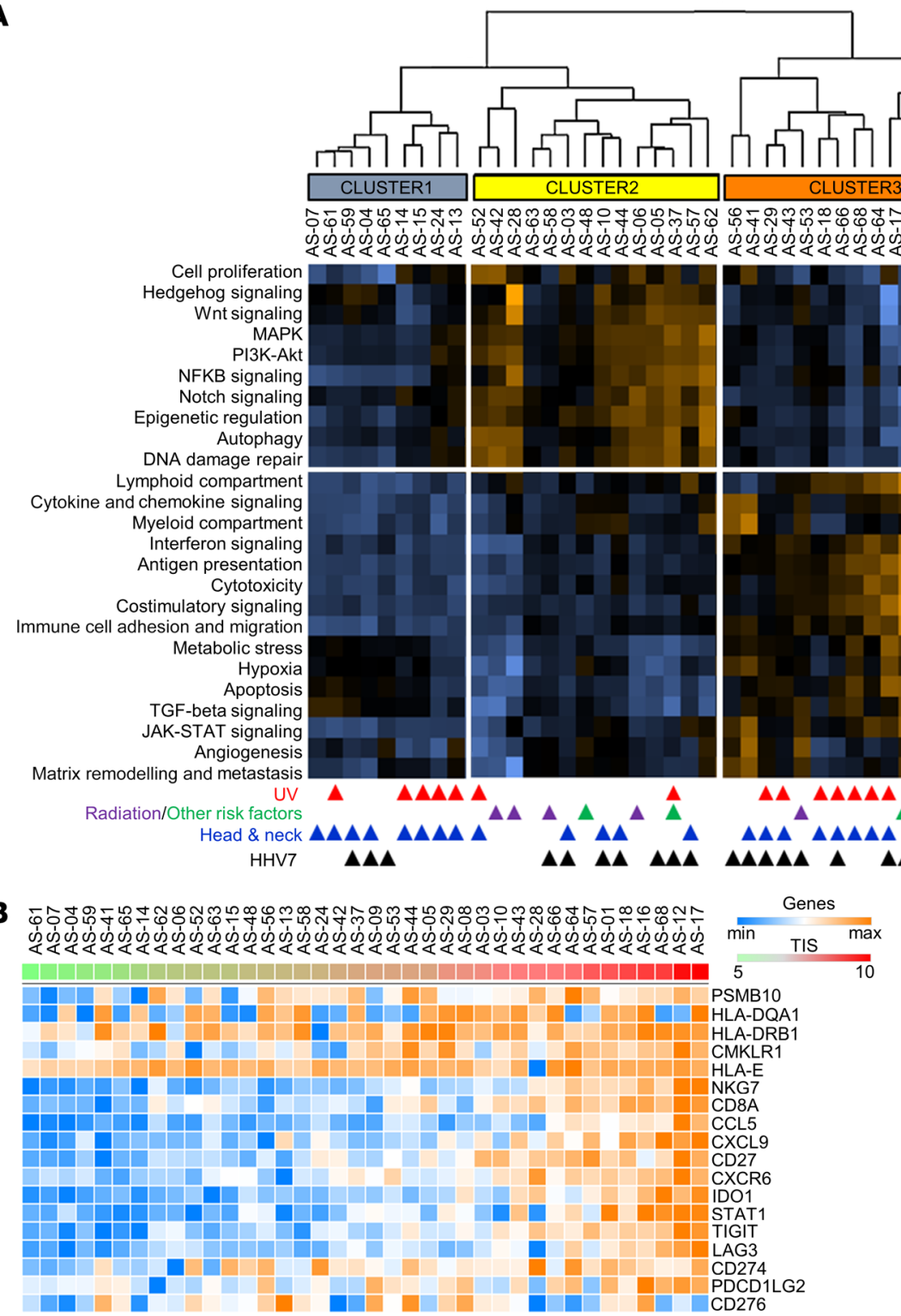

Figure 2. Unique alterations of immune, microenvironmental, and tumor-related pathways define distinct clusters of angiosarcoma. (A) On the NanoString 10360 panel, 3 distinct clusters were identified on the basis of similarities in clinical features, pathway scores, and immune profiles. Clusters 1 and 2 were characterized by a relative lack of immune-related signaling and immune cells as compared with cluster 3. (B) TIS scores, with a median of 6.76 (range, 5.23-9.65). max, maximum; min minimum. (C) TIS scores were highest in cluster 3 compared with clusters 2 and 1 (7.54 vs. 6.71 vs. 5.75, respectively: Kruskal-Wallis, $P<0.0001$ ). (D) Clusters 1 and 3 comprised mostly primary head and neck angiosarcomas (Fisher's exact, $P=0.0138)$, $(E)$ frequently with evidence of UV-related DNA damage (Fisher's exact, $P=0.0196$ ). (F) Cluster 2 exhibited significant expression levels of DNMT1, MYC, and HRAS, in keeping with upregulation of epigenetic and oncogenic signaling pathways.
D

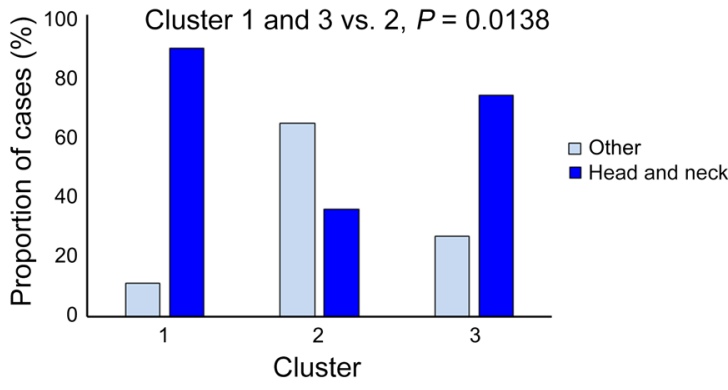

E

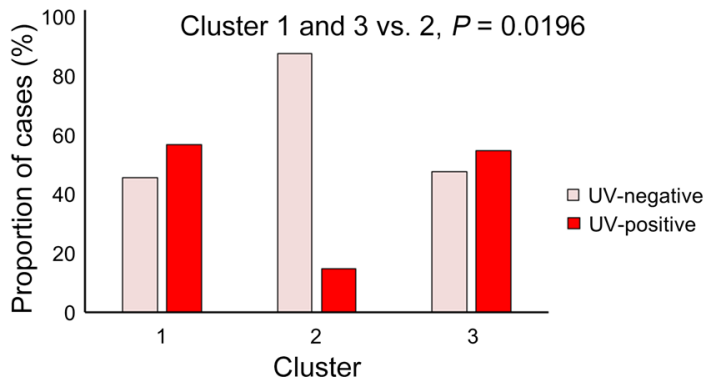

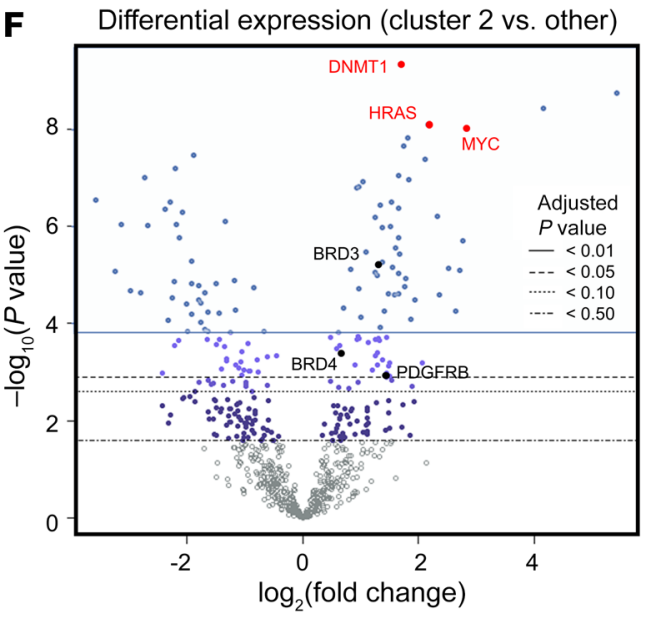

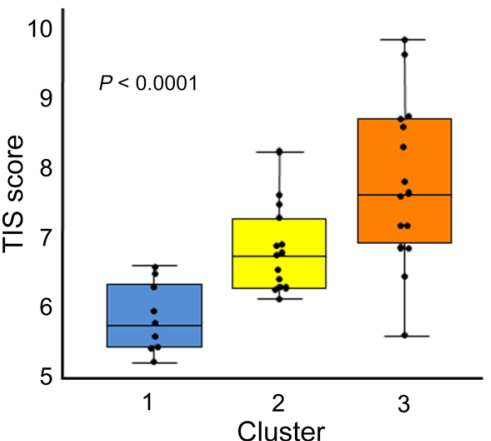


available. Differentially expressed genes in tumor-normal paired samples are summarized in Supplemental Table 5. On gene set enrichment analysis (GSEA), the top-scoring gene sets included those involved in cell cycle-related and inflammation-related pathways (Figures 3, A and B, and Supplemental Figures 8 and 9). The top 3 highest-scoring cell cycle-related gene sets included "E2F targets" (normalized enrichment score [NES] = 2.78, FDR $Q<0.001, P<0.001)$, "G $\mathrm{M}_{2}$ checkpoint" $(\mathrm{NES}=2.64, \mathrm{FDR} Q<$ $0.001, P<0.001)$, and "mitotic spindle" $(\mathrm{NES}=2.06$, FDR $Q<$ $0.001, P<0.001)$; the top 3 highest-scoring inflammation-related gene sets included "allograft rejection" $(\mathrm{NES}=2.70$, FDR $Q$ $<0.001, P<0.001)$, “interferon gamma response" $(\mathrm{NES}=2.58$, FDR $Q<0.001, P<0.001$ ), and "interferon alpha response" (NES $=2.35$, FDR $Q<0.001, P<0.001)$ (Supplemental Tables 6 and 7).

Notably, among the core enriched genes in the E2F and other cell cycle-related gene sets, we identified several therapeutically targetable kinases including AURKA, AURKB, PLK1, PLK4, CHEK1, and CDK4 ( $\log _{2}$ fold change 1.30, 3.15, 2.05, 1.85, 1.40, 0.79 , respectively) (Supplemental Table 8). We observed overexpression of these genes across the tumors and cell lines compared with expression in normal tissue (all $P<0.01$ by Mann-Whitney $U$ test) (Figure 3C). Expression of these kinases was generally correlated with selected genes (HRAS, MYC, and DNMT1) that were upregulated in cluster 2 (as identified from NanoString analyses), supporting a major role of oncogenic signaling in this subset of angiosarcomas (Figure 3D).

In both angiosarcoma cell lines, treatment with aurora kinase inhibitors (alisertib, tozasertib, barasertib), polo-like kinase inhibitors (volasertib, GSK461364), and CHK1 inhibitors (AZD7762, prexasertib, GDC-0575) resulted in markedly reduced viability in a dose-dependent manner (Figure 3E). Remarkably, we found that the compounds tested were highly potent, achieving an $\mathrm{IC}_{50}$ below $1 \mu \mathrm{M}$ in at least 1 compound per class in both cell lines. In contrast, the same compounds were significantly less potent in nonmalignant endothelial cells (Supplemental Figure 10). Treatment with the CDK4/6 inhibitor palbociclib, however, was ineffective in both cell lines $\left(\mathrm{IC}_{50}>10 \mu \mathrm{M}\right)$ (Supplemental Figure 10).

Immune cell composition and $\mathrm{mIHC} / \mathrm{IF}$. In order to computationally infer immune cell-type enrichments in angiosarcoma and matched normal tissue samples, we used the CIBERSORTx tool (https://cibersortx.stanford.edu/) to analyze bulk transcriptomic data (Supplemental Table 9). The predominant cell types were macrophages followed by $\mathrm{CD} 4^{+} \mathrm{T}$ cells. Overall, we observed a relative enrichment in immune cells in tumor tissue versus matched normal tissue (median score 0.99 vs. $0.57, P=0.0097$ ). Across tumor samples, we observed a significant range of absolute scores (0.57-1.37), implying intertumoral heterogeneity of infiltrating immune cells (Figure 4).

In order to further dissect the immune cell composition of angiosarcoma, we inferred immune cell types using NanoString gene expression data. As expected, we found that cluster 3 tumors were strongly enriched for most immune cell types, except Th1 cells, mast cells, and B cells (Figure 5A). Compared with those within clusters 1 and $2, \mathrm{UV}^{+}$tumors in cluster 3 were enriched for most immune cell types, whereas $\mathrm{UV}^{-}$tumors were only enriched for neutrophils and macrophages (Figure 5B). Using multiplex immunohistochemistry/immunofluorescence (mIHC/IF) to visualize and quantitate tumor-infiltrating immune cells, we observed that the proportion of neutrophils $\left(\mathrm{CD} 15^{+}\right)$, macrophages $\left(\mathrm{CD}^{\circ} 8^{+}\right)$, cytotoxic T cells $\left(\mathrm{CD}^{+}\right)$, Tregs $\left(\mathrm{FOXP}^{+}\right)$, and $\mathrm{PD}-\mathrm{L1}^{+}$cells relative to tumor cells $\left(\mathrm{ERG}^{+}\right)$correlated directly with the corresponding cell type scores inferred from NanoString transcriptomic profiling (Figure 5, C and D). All immune cell infiltrates were enriched in cluster 3 compared with clusters 2 and 1 . We also observed that PD-L1 ${ }^{+}$macrophages, but not PD-L1 ${ }^{+}$ tumor cells, were significantly enriched in cluster 3 tumors (Supplemental Figure 11).

Metagenomic detection of $H H V-7$ in human angiosarcoma. Incidentally, on screening for the presence of potential angiosarcoma-associated viruses, we detected viral reads mapping to the human herpesvirus 7 (HHV-7) genome (NCBI: gi_51874225_ref_ NC_001716.2) in the tumor tissue of patient AS-01 but not the adjacent matched normal component. The assembled viral contigs were aligned back to the HHV-7 reference genome with an identity of $99.80 \%$ (Figure 6A). None of the sequenced libraries suggested the presence of any integrated virus. We verified the presence of HHV-7 in patient AS-01 by PCR and IHC (Figure 6B). We could not detect actively transcribed viral genes at the transcriptomic level, supporting a latent state of the virus. In the overall cohort, we detected HHV-7 in 44 of 68 patients $(64.7 \%)$ (Supplemental Table 10), but the virus was detected at a lower frequency in an independent cohort of angiosarcoma samples from a Western cohort (4 of 17 patients, 23.5\%) (Supplemental Table 11).

Interestingly, in an exploratory GSEA using the cases profiled with RNA-Seq (Supplemental Figure 13), we observed that HHV-7 ${ }^{+}$ angiosarcomas were associated with the expression of genes involved in inflammation-related pathways, whereas cell cyclerelated pathway genes were enriched in virus-negative tumors (Figure 6C, Supplemental Figure 12A, and Supplemental Tables 12 and 13). Additionally, we observed an inverse correlation between HHV-7 positivity and the presence of UV-related mutational signatures. HHV $-7^{+}$angiosarcomas had lower levels of $\mathrm{C}>\mathrm{T}$ mononucleotide substitutions $(P=0.0333)$ and $\mathrm{CC}>\mathrm{TT}$ dinucleotide substitutions $(P=0.0127)$ (Figure 6D). Corroborating the analysis from RNA-Seq, NanoString profiling revealed that genes involved in IFN and other immune signaling pathways were markedly enriched in $\mathrm{HHV}-7^{+}$tumors (Supplemental Figure 12B). Compared with $\mathrm{HHV}-7^{-}$angiosarcomas, $\mathrm{HHV}-7^{+}$tumors were associated with high TIS scores (66.7\% vs. 29.4\%, $P=0.0242)$ and were overrepresented in cluster $3(P=0.0497)$ (Figure 6E). Virus-positive tumors were strongly enriched for the myeloid compartment, with top cell-type scores for mast cells, macrophages, and neutrophils (Figure 6F). The significance of HHV-7 in angiosarcomas will require further investigation and validation.

\section{Discussion}

Modern molecular methods including next-generation sequencing have only recently begun to characterize the pathobiology of angiosarcoma, an otherwise poorly understood disease with an aggressive clinical phenotype regardless of ethnogeographic differences $(2,3)$. We have noticed that cutaneous angiosarcomas of the head and neck region are more prevalent in Asian than Western patients, raising the possibility of unique genetic or environmental factors influencing its pathogenesis (3). Previously, recur- 
A

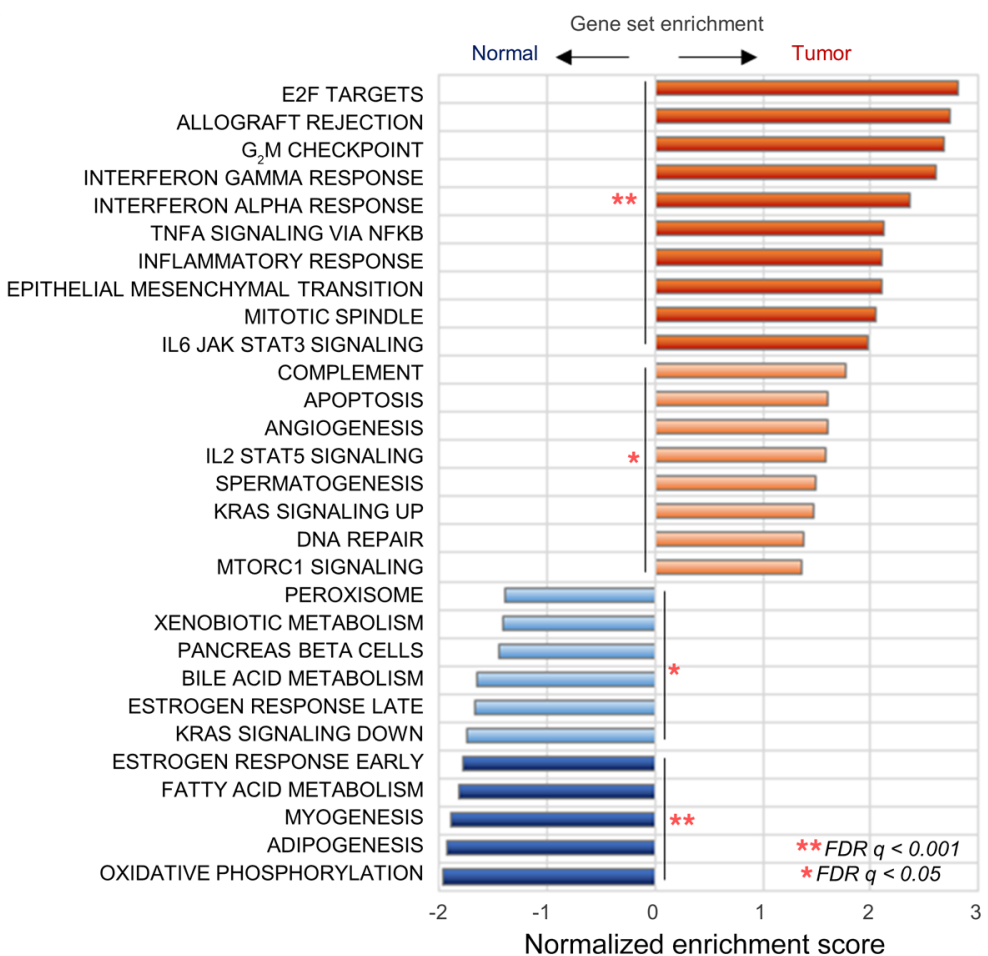

B
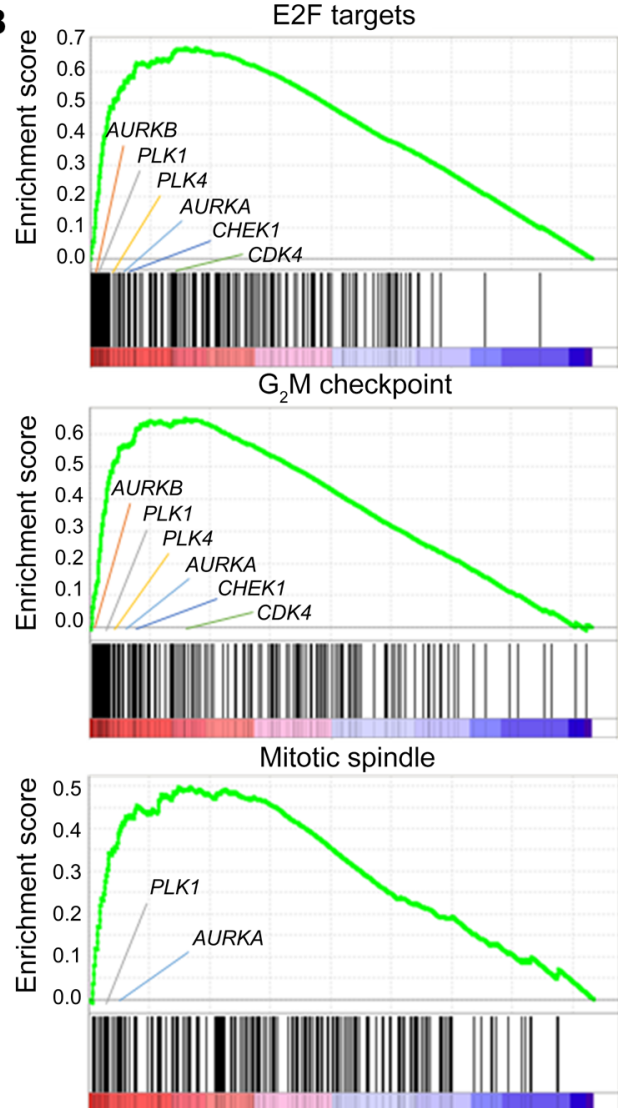

C

$P<0.01$ for all genes tumor vs. matched normal

을 $12=A U R K A=A U R K B=P L K 1=P L K 4=C H E K 1=C D K 4$

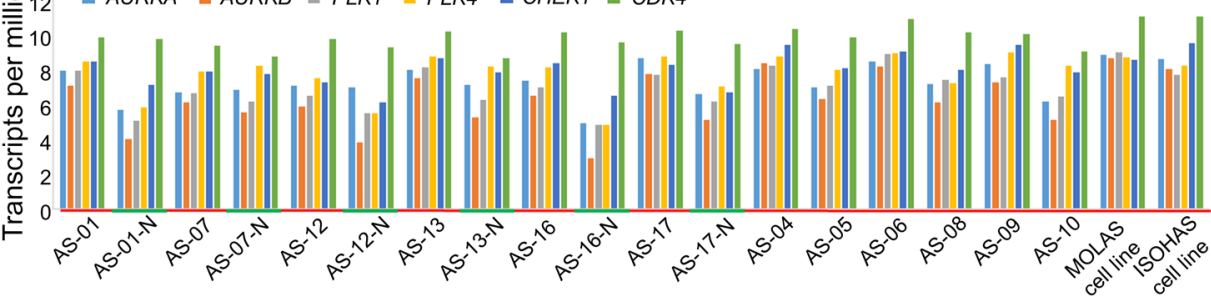

D

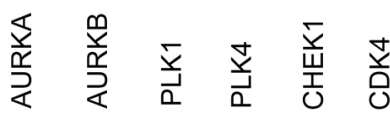

HRAS 0.70270 .66610 .84870 .58330 .71400 .6173

MYC 0.52900 .66950 .82710 .43750 .50000 .7491

DNMT1 0.77340 .81330 .69520 .54070 .43750 .6110

E

MOLAS

cell line


Concentration (nM)
ISOHAS cell line



Concentration (nM)

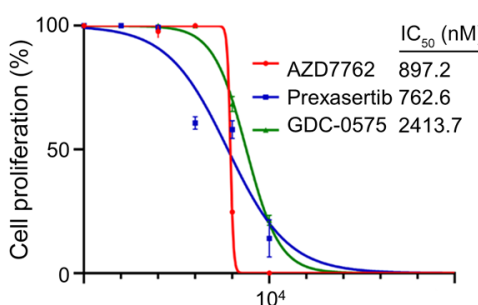

Concentration (nM)
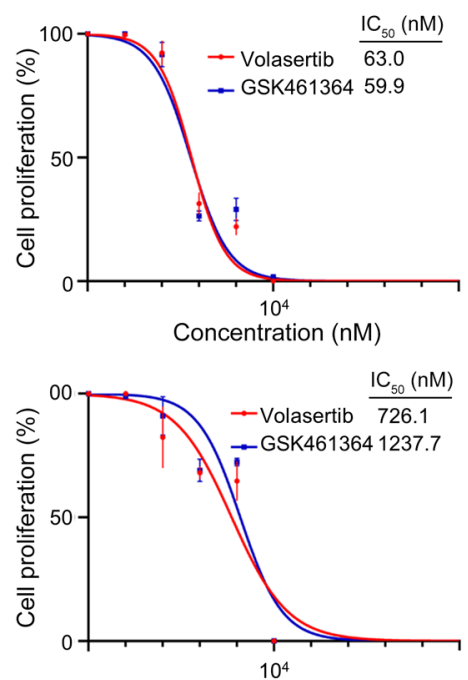

Concentration (nM) 
Figure 3. Enrichment of distinct and actionable genetic pathways in angiosarcoma. (A) GSEA highlighted top-scoring gene sets in cell cyclerelated and inflammation-related pathways in the tumor compartment. (B) The top 3 highest-scoring cell cycle-related gene sets include "E2F targets" (NES $=2.78$, FDR $\underline{0}<0.001, P<0.001$ ), " $\mathrm{C}_{2} \mathrm{M}$ checkpoint" (NES = 2.64, FDR $\underline{Q}<0.001, P<0.001$ ), and "mitotic spindle" (NES = 2.06, FDR $\underline{Q}$ $<0.001, P<0.001)$. (C) Among the core enriched genes, several therapeutically targetable kinases were identified, including AURKA, AURKB, PLK1, $P L K 4, C H E K 1$, and CDK4. Overexpression of these genes was observed across the tumors and cell lines (MOLAS and ISOHAS) compared with expression in normal tissue. (D) Expression of these kinases were generally correlated with selected genes (HRAS, MYC, and DNMT1) that were upregulated in cluster 2 (as identified from NanoString analyses). Pearson's coefficient values are shown. (E) In angiosarcoma cell lines, treatment with aurora kinase inhibitors (alisertib, tozasertib, barasertib), polo-like kinase inhibitors (volasertib, GSK461364), and CHK1 inhibitors (AZD7762, prexasertib, GDC-0575) resulted in markedly reduced viability in a dose-dependent manner. All drug treatments were performed in triplicate, and results are represented by mean values and SDs.

rent somatic mutations of angiogenic signaling pathway genes, such as KDR, PTPRB, and PLCG1, have been identified in more than $40 \%$ of patients with angiosarcoma $(5,6,8,12,13)$. Mutations of genes involved in the MAPK, PIK3CA/AKT/mTOR, and TP53 signaling pathways have been implicated as well $(14,15)$. Mutation of POT1, a gene involved in the regulation of chromosome stability and telomerase activity, was detected in TP53- Li-Fraumeni-like families with cardiac angiosarcoma (16). Our results confirm the presence of several of these somatic alterations in angiosarcomas. Additionally, in line with recent findings from the ASCproject, we observed a subgroup of cutaneous head and neck angiosarcomas with higher TMB and dominant UV signatures. This observation provides a biological rationale for the use of therapeutic immune checkpoint inhibition in this group of tumors - a hypothesis supported by exceptional responses in selected patients (10).

Intriguingly, we observed that only approximately half of all head and neck angiosarcomas showed significant UV damage, whereas the others were mutationally quiet and non-UV driven. This subgroup of cutaneous head and neck angiosarcomas without dominant UV signatures was also observed, albeit at a lower frequency, in 2 of 10 patients (20\%) from the ASCproject (10). Collectively, these findings indicate a genomic heterogeneity of angiosarcomas, even within those of similar (head and neck) origins. Although the technical differences and small sample sizes in both studies may account for such a difference, it may be speculated that unique ethnogeographic or etiologic factors (e.g., viral) may exist within Asian and Western populations.

Our transcriptomic profiling results identified several actionable protein kinases in angiosarcoma, the efficacy of which was confirmed in vitro. These results are in keeping with previously reported objective responses in patients with angiosarcoma who were treated with the aurora kinase A) inhibitor alisertib (17), and support the investigation of the other targets in prospective clinical trials. In addition, the identification of cluster 2 angiosarcomas with upregulation of epigenetic and oncogenic signaling pathway genes is intriguing, and further studies should be performed on the potential use of epigenetic drugs including DNMT or BET inhibitors, or agents targeting PDGFR $\beta$ such as imatinib (18-20). A previous study by Styring et al. investigated gene expression using the Illumina BeadArray platform in secondary angiosarcomas of the breast and similarly demonstrated the upregulation of genes involved in oncogenic signaling including MYC, KIT, and RET (21). The study by Styring et al. supports our finding that cluster 2 as a unique entity defined by enrichment for secondary angiosarcomas and has a distinct gene expression signature.

More interesting perhaps, with relevance to immunotherapy, is our finding of enriched inflammation-related pathways and immune cells in a subset of angiosarcomas. Specifically, we identified a distinct cluster (cluster 3) represented by overexpression of inflammation- and immune-related signaling pathway genes, as well as strong enrichment for immune cells. TIS scores were highest within this cluster, suggesting that this subgroup of angiosarcomas may benefit from checkpoint immunotherapy (22). A dissection of the cases by etiology and anatomical location revealed interesting disease patterns. UV-related angiosarcomas as well as those arising from the head and neck were predominantly found in clusters 1 and 3. Collectively, the data support heterogeneity in immune and mutational profiles of angiosarcoma, particularly those arising in the head and neck. The clinical implications of such findings are interesting, given recent reports that both high TMB and TIS scores independently conferred higher response rates to immune checkpoint inhibitors across various tumor types (22-24), therefore supporting the need for comprehensive interrogation of angiosarcomas using a multiomic approach. Nonetheless, it remains to be validated whether angiosarcomas concurrently harboring a high TMB (as a result of UV mutagenesis) and an inflamed phenotype will show the best responses to immune checkpoint inhibition. On the other hand, by using mIHC/IF, we validated our genetic findings at the protein level by quantifying immune cell subsets such as $\mathrm{CD} 8^{+} \mathrm{T}$ cells, $\mathrm{CD} 68^{+}$macrophages, $\mathrm{FOXP}^{+}$Tregs, and $\mathrm{CD}^{+} 5^{+}$neutrophils in the angiosarcoma tissue samples in this cohort. In a direct correlation with the NanoStringderived data sets, all immune cell subsets were enriched in cluster 3 compared with clusters 2 and 1 . $\mathrm{mIHC} / \mathrm{IF}$ is a powerful tool to study the spatial tumor microenvironment of limited tissue specimens (25-31) and has shown great potential in clinical and translational applications $(32,33)$. mIHC/IF, which allows for staining of up to 6 markers on a single slide, allows a broader range of analysis compared with IHC. The reproducibility of this technique and correlation with conventional IHC have been reported by our group and others for various markers, including some of those used in this study, such as CD8, CD68, FOXP3, and PD-L1 (3437). Moreover, mIHC/IF workflow has been partially automated from staining to analysis (38), reducing hands-on time. Recently, Lu et al. reported that their analysis of data from 10 different solid tumor types from 8135 patients revealed that $\mathrm{mIHC} / \mathrm{IF}$ is a superior modality for predicting the response to anti-PD-1/antiPD-L1 treatment compared with TMB, gene expression profile (GEP), and conventional IHC scoring for PD-L1 (31). We believe that, upon validation in a larger cohort, our IHC-based techniques might be easily translated to routine clinical practice for the management of angiosarcoma.

Our exploratory attempt to discover potential angiosarcomarelated viruses via analysis of the viral metagenome led to the identification of HHV-7, a betaherpesvirus virus best known to be associated with a benign exanthem known as pityriasis rosea 



Figure 4. Immune cell type abundance in angiosarcoma. (A) CIBERSORTx was used to computationally infer immune cell type enrichments in angiosarcoma and matched normal tissue samples. The predominant cell types were macrophages followed by CD4+ $T$ cells. Overall, a relative enrichment was observed in immune cells in tumor versus matched normal tissue (median score 0.99 vs. $0.57, P=0.0097$, Mann-Whitney $U$ test). Across tumor samples, a significant range of absolute scores (0.57-1.37) was observed, implying an intertumoral heterogeneity of infiltrating immune cells. (B) In most cases, a relative enrichment in immune subpopulations was observed in tumor tissue versus matched normal tissue.

(39). The exact significance of our finding and the contribution of HHV-7 to the pathogenesis of angiosarcoma remain to be further investigated, although this virus was detected in two-thirds of our angiosarcoma cohort and was predominantly present in non-UV-related cases. Our results from the delineation of transcriptomic profiles by virus status revealed that HHV-7 may drive inflammation-related pathways, including those involved in the IFN response. Compared with HHV-7- tumors, we found that $\mathrm{HHV}-7^{+}$angiosarcomas were associated with high TIS scores and were overrepresented in cluster 3 . We speculate that, in subsets of angiosarcomas infected with HHV-7, the viral antigens trigger a heightened adaptive immune response that subsequently becomes exhausted. Furthermore, HHV-7 may contribute to angiosarcoma development through an immunomodulatory mechanism. Like all herpesviruses, HHV-7 persists lifelong following infection of the host cell (40). In order to avoid detection and elimination by the host immune system, HHV-7 may evoke several immune escape mechanisms in the infected host cell, including downregulation of HLA class I and II and $\beta$ 2-microglobulin expression in infected cells, thereby preventing recognition of these markers by cytotox- 
ic T lymphocytes (41). In particular, this process has been shown to be mediated by the HHV-7 immunoevasin U21, which diverts MHC class I molecules from the ER to the lysosomal compartments, resulting in the premature degradation of these molecules (42). Further studies are underway to better characterize the role of this virus in angiosarcoma development.

In conclusion, the current study suggests the existence of angiosarcoma subtypes characterized by distinct etiological and biological phenotypes, opening up opportunities for improved prognosis and treatment of this rare disease and potentially proving useful for patient selection in clinical trials. Further studies of larger independent data sets should be conducted to validate these observations.

\section{Methods}

Patient cohort. Sixty-eight patients diagnosed with angiosarcoma at the Singapore General Hospital (SGH) and National Cancer Centre Singapore (NCCS) between January 2000 and December 2015 were identified. Snap-frozen tissue samples from 18 patients were available, and 13 of these samples included paired normal tissue or blood and were sent for whole-genome sequencing. Archival formalin-fixed, paraffin-embedded (FFPE) angiosarcoma samples from all 68 patients were available. Genetic material isolated from snap-frozen tissue was available from an independent cohort of patients with angiosarcoma $(n=17)$ from the MD Anderson Cancer Center (MDACC). The clinicopathological characteristics of all patients with angiosarcoma and the profiling methods applied to the study cohort are summarized in Supplemental Table 10 and Supplemental Figure 13.

Cell lines. The SUP-T1 immature T lymphocyte cell line latently infected with HHV-7 (JI strain) was maintained in RPMI 1640 medium supplemented with $10 \%$ FBS, $1 \%$ penicillin-streptomycin, and $2 \mathrm{mM}$ L-glutamine. This cell line was derived from malignant cells collected from the pleural effusion of an 8-year-old child with T cell lymphoblastic lymphoma (courtesy of Dharam Ablashi, NIH AIDS Reagent Program, Division of AIDS, NIAID, NIH). The uninfected SUP-T1 cell line was obtained from the American Type Culture Collection (ATCC). Two angiosarcoma cell lines (MO-LAS-B and ISOHAS-B) were obtained from the Cell Resource Center for Biomedical Research, Institute of Development, Aging and Cancer (Tohoku University, Sendai, Japan), courtesy of Mikio Masuzawa (Kitasato University, Tokyo, Japan). The MO-LAS-B cell line was established from a patient with metastatic scalp lymphangiosarcoma to the pleura, whereas the ISO-HAS-B cell line was established from a patient with primary scalp hemangiosarcoma. Both cell lines were maintained in DMEM medium supplemented with $10 \%$ FBS and $1 \%$ penicillin-streptomycin. Authentication of both cell lines was performed using 24 short tandem repeat (STR) loci plus the sex-determining locus, amelogenin, amplified using the GenePrint 24 System from Promega (Axil Scientific Pte Ltd.) (Supplemental Table 14). CD31 immunostaining was also performed, and cellular growth was verified in culture (Supplemental Figure 10). Primary human dermal microvascular endothelial cells (HDMECs) were obtained from PromoCell. Cell cultures at approximately $70 \%$ confluence were used for all experimental drug treatments unless otherwise stated.

Whole-genome sequencing and viral genome identification. The whole genomes of snap-frozen tumor tissue with adequate tumor content were selected for whole-genome sequencing (paired tumor-normal samples, $n=13$; tumor-only samples, $n=5$ ). A qualified pathologist performed the initial microscopic evaluation and assessment of tumor content. Whole-genome sequencing was performed on the Illumina HiSeq $\mathrm{X}$ platform as paired-end 150 bp reads, using DNA inserts averaging 350 bp (NovogeneAIT Genomics) (Supplemental Table 15). Read pairs were aligned to the human reference genome NCBI GRC Build 37 (hg19) using Burrows-Wheeler Aligner-MEM (BWA-MEM) (http://bio-bwa. sourceforge.net) (43), and SAMBamba (44) was used to remove PCR duplicates. In order to identify viral genomes, an alignment-based analysis was carried out using VirusFinder 2 (45), and a subroutine, using Trinity, assembled putative viral short reads to longer contigs (46).

Somatic variant calling and generation of mutation signatures. Somatic mutations were identified by the Strelka2 variant caller with default parameters (47). Variants were subsequently annotated by WANNOVAR (48). TMB was estimated on the basis of the proportion of nonsynonymous single nucleotide variants and short indels per coding megabase. Somatic mutational signatures were extracted using SigProfiler, an algorithm based on the 96 base substitution classification via nonnegative matrix factorization, and compared with the COSMIC v3 set of signatures (49). Dinucleotide substitutions were identified as previously described (50). Manta SV was used to detect structural variants (51). GISTIC 2.0 was used to identify biologically significant copy number changes from random events (52).

cDNA synthesis, whole transcriptome sequencing, and GSEA. Total RNA was extracted from snap-frozen tissue using TRIzol (Invitrogen, Thermo Fisher Scientific) and purified with an RNeasy Mini Kit (QIAGEN) according to the manufacturer's instructions. The integrity of RNA was determined by electrophoresis using the 2100 Bioanalyzer (Agilent Technologies). Whole-transcriptome sequencing (WTS) was performed on the Illumina HiSeq4000 platform (AuraGen) using the standard Illumina RNA-Seq protocol (paired tumor-normal samples, $n=6$; tumor-only samples, $n=6$; cell lines, $n=2$ ) (Supplemental Table 16). The reads were aligned to the human genome hg19 RefSeq reference transcriptome by $\operatorname{STAR}(53,54)$. Transcript abundance estimation was performed using RSEM software (55). Differentially expressed genes were identified using DESeq2 (56). For each gene, read counts are represented as transcripts per million (TPM) and are normalized for both sequencing depth and gene length. GSEA was performed using the Molecular Signatures Database (MSigDB) hallmark gene set (57). A gene set is significantly enriched if its NES has a FDR $Q$ value below 0.05. Immune cell type abundance from bulk transcriptomes were inferred with CIBERSORTx using LM22 immune reference profiles (58).

NanoString gene expression profiling. The NanoString Pancancer IO360 panel on the nCounter platform (NanoString Technologies) was used to interrogate gene expression on FFPE tissue, following the manufacturer's protocol. Briefly, RNA was extracted from five $10 \mu \mathrm{m}$ sections on all samples with adequate tumor tissue available and then analyzed using the 2100 Bioanalyzer (Agilent Technologies). After excluding samples with suboptimal RNA integrity and content, the remaining samples were included in the nCounter analysis. The final set of data that passed quality control $(n=38)$ were analyzed on the nSolver 4.0 Advanced Analysis module using default settings to derive differentially expressed genes, pathway scores, and cell type scores. The panel was further analyzed using the TIS algorithm, which measures the level of immune infiltrate in a tumor and the tumor microenvironment (11). This signature contains 18 genes related to antigen 
A



$\mathrm{CD} 8^{+}$

$\mathrm{T}$ cells

C
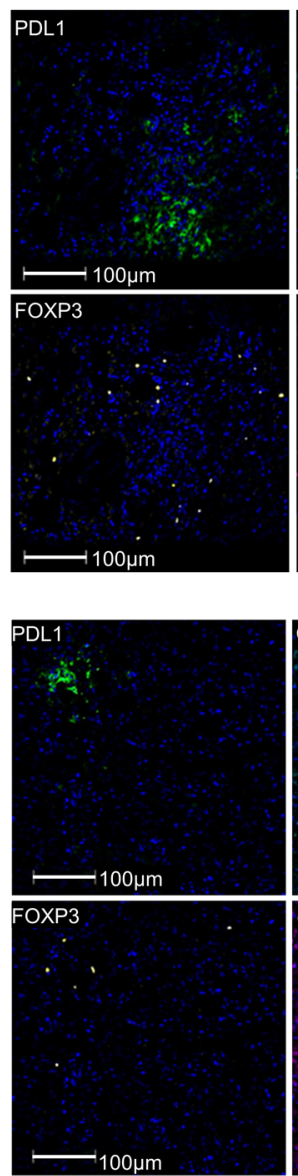

AS-16 (cluster 3)
Th1 cells
Mast cells

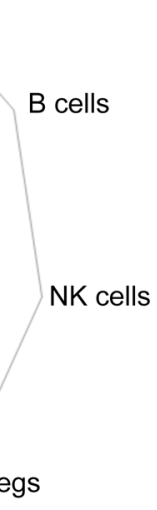

B

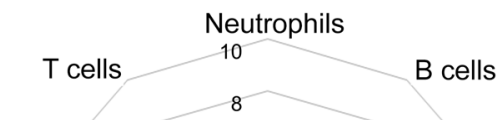

Cytotoxic cells

$\mathrm{CD}^{+}$

T cells

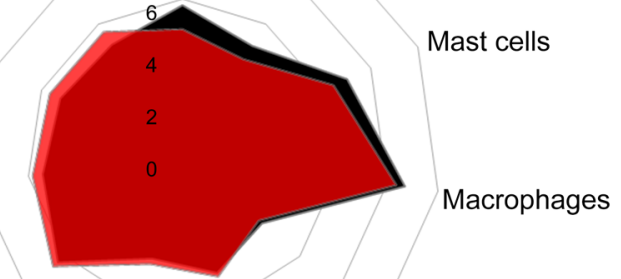

CD45

cells

Th1 cells
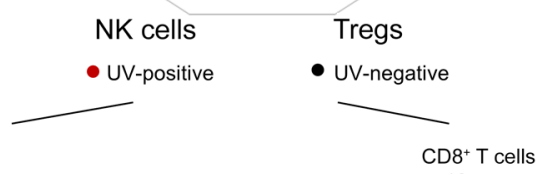

T cells Neutrophils

- Cluster 3

- Cluster $1 / 2$

Cytotoxic cells

CD45 cells



Neutrophils

10 Mast cells

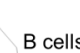

Neutrophils

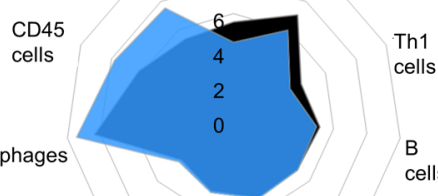

Mast cells

NK cells

T cells

Th1 cells

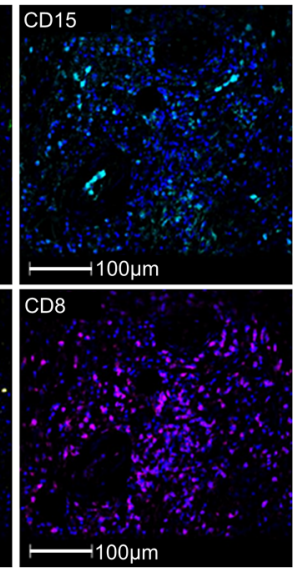

AS-13 (cluster 1)
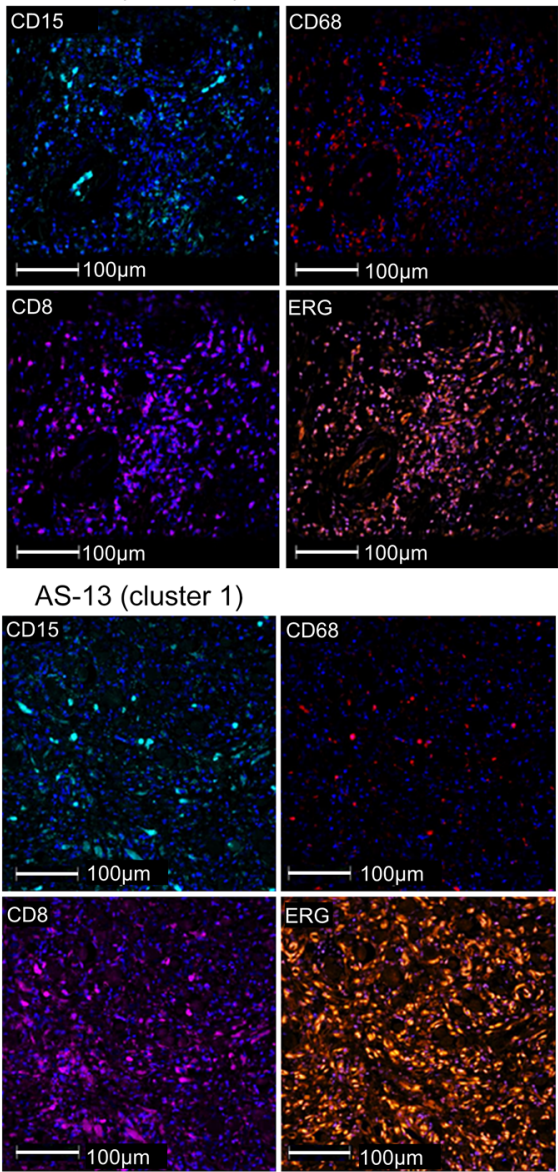
- Cluster 3

- Cluster $1 / 2$

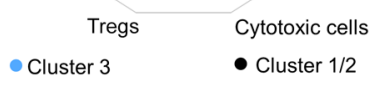

D
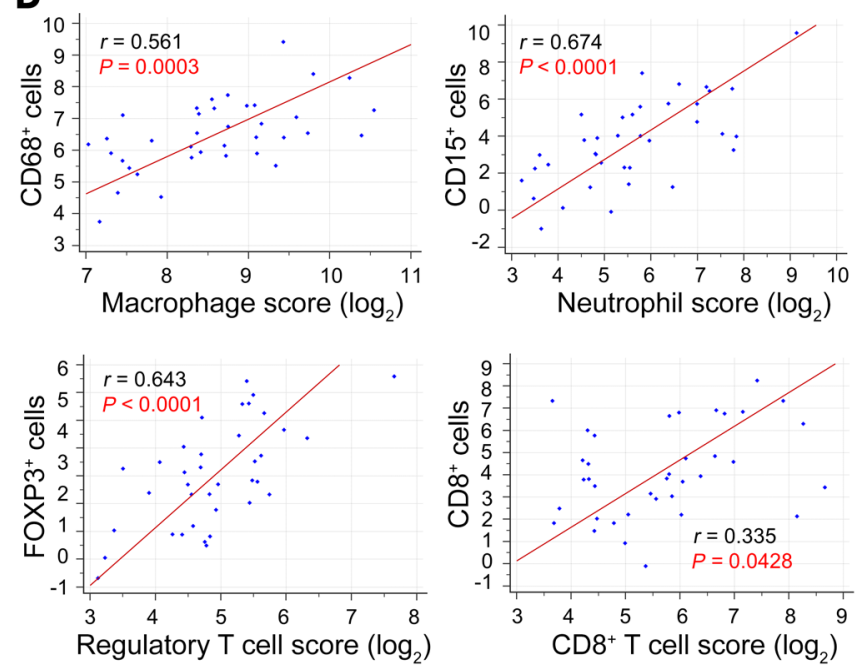

ํํ 1

CD8 ${ }^{+} \mathrm{T}$ cell score $\left(\log _{2}\right)$ (n)

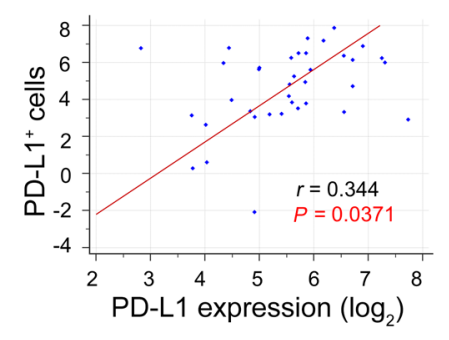


Figure 5. Verification of tumor-infiltrating immune cells using $\mathrm{mIHC}$ / IF. (A) As inferred using NanoString gene expression data, cluster 3 tumors were strongly enriched for most immune cell types, except Th1 cells, mast cells, and B cells. (B) Compared with those within clusters 1 and $2, \mathrm{UV}^{+}$tumors in cluster 3 were enriched for most immune cell types, whereas UV' tumors were only enriched for neutrophils and macrophages. (C) Representative mIHC/IF images of immune cells in samples AS-16 (cluster 3) and AS-13 (cluster 1). Scale bars: $100 \mu \mathrm{m}$. (D) The proportion of neutrophils (CD15+), macrophages (CD68 $8^{+}$, cytotoxic T cells (CD8+), and Tregs $\left(\mathrm{FOXP3}^{+}\right)$relative to tumor cells $\left(\mathrm{ERG}^{+}\right)$correlated directly with the corresponding cell type scores inferred from NanoString transcriptomic profiling. Pearson's coefficients with corresponding $P$ values are shown.

presentation, chemokine expression, cytotoxic activity, and adaptive immune resistance. The score was calculated as a weighted linear combination of the expression values of the 18 genes normalized to expression of the stable housekeeping gene. A high TIS score was defined as being greater than or equal to the median score in the cohort.

HHV-7 nested PCR. Nested PCR was performed to detect HHV-7 DNA sequences targeting the U10 (structural phosphoprotein) gene. For positive controls, HHV-7 was detected via PCR for the U10 gene using DNA isolated from SUP-T1 cell lines latently infected with HHV7. The specificities of these primers were checked against HHV-7 JI and RK strains by Primer-Blast and by direct sequencing of the PCR products (Supplemental Table 17). The PCR cycling conditions were as follows: initial denaturation for 2 minutes at $94^{\circ} \mathrm{C}$, followed by 35 cycles of denaturation for 30 seconds at $94^{\circ} \mathrm{C}$, annealing for $30 \mathrm{sec}$ onds at $60^{\circ} \mathrm{C}\left(55^{\circ} \mathrm{C}\right.$ for inner reaction), and extension for 45 seconds at $72^{\circ} \mathrm{C}$, ending with a final extension step for 5 minutes at $72^{\circ} \mathrm{C}$. The reaction mixture with a final volume of $25 \mu \mathrm{L}$ consisted of $100 \mathrm{ng}$ DNA template, $1.5 \mathrm{mM} \mathrm{MgCl}_{2}, 0.2 \mathrm{mM} \mathrm{dNTP}, 0.5 \mu \mathrm{M}$ primer, and 2 units Taq polymerase. The PCR product $(0.5 \mu \mathrm{L})$ from the first round of amplification was used as the template for the second round of PCR. The conditions were the same as for the first PCR except that the inner primers were used instead of the outer primers. For the positive control, DNA extracted from an HHV-7-infected SUP-T1 cell line was used, whereas for negative controls, DNA extracted from an HHV-7uninfected SUP-T1 cell line as well as reactions with no DNA template were used. Amplified PCR products were electrophoresed within $1.5 \%$ agarose gels and visualized with UV light fluorescence after SYBR safe staining. To avoid contamination, DNA extraction, PCR, and gel electrophoresis were performed in separate laboratory locations using separate sets of equipment.

IHC. Sections ( $4 \mu \mathrm{m}$ thick) were cut from the FFPE tissue blocks and mounted onto positively charged Bond Plus Slides (Leica Biosystems) and then dried on a heating bench for at least 20 minutes. After deparaffinization and rehydration, tissue samples were subjected to high temperature-induced epitope retrieval by briefly steaming them in antigen retrieval solution (citrate buffer, $\mathrm{pH}$ 6.0) for 3 minutes in a pressure cooker. Endogenous peroxidase activity was blocked using BLOXALL Blocking Solution (Vector Laboratories) for 10 minutes at room temperature, followed by $2.5 \%$ normal horse serum blocking solution for 20 minutes, and then blocking of endogenous biotin or avidin/streptavidin binding proteins using the Avidin/Biotin Blocking Kit (Vector Laboratories). Using the VECTASTAIN Elite ABC-Peroxidase Kit (Vector Laboratories), primary antibodies were diluted at $1: 200$ in $2.5 \%$ normal horse serum. Slides were incubated for 30 minutes each at room temperature in sequence with the respective primary and secondary antibodies, followed by ABC Reagent (Vector Laboratories). Substrate detection was performed using the Vector NovaRED Substrate Kit (Vector Laboratories), before counterstaining briefly with Vector Hematoxylin QS Counterstain (Vector Laboratories). Mouse monoclonal IgG1 antibody (BioAcademia) recognizing the HHV-7 glycoprotein gH (clone 2) was used to detect the presence of HHV-7. Mouse monoclonal IgG2 antibody (clone TDM2, Cosmo Bio USA) was used to detect CPDs (Cosmo Bio USA). Tumor cells with moderate-to-intense staining for HHV-7 or CPDs were regarded as positive, whereas weak/equivocal cases were regarded as negative.

$m I H C / I F . m I H C / I F$ was performed using an Opal Multiplex IHC Kit (Akoya Bioscience), as previously described by our group and in other studies (59-68). All cases profiled on NanoString were available except for AS-15 and are included in this analysis $(n=37)$. Slides were labeled with PD-L1, CD68, CD8, FOXP3, CD15, and ERG, followed by the appropriate secondary antibodies. Particularly for this panel, we followed the detailed protocol that our group previously reported (38) and have briefly described here. FFPE tissue sections were cut onto Bond Plus slides (Leica Biosystems) and heated at $60^{\circ} \mathrm{C}$ for 20 minutes (69). Tissue slides were then subjected to deparaffinization, rehydration, and heat-induced epitope retrieval (HIER) using a Leica Bond Max Autostainer (Leica Biosystems), before endogenous peroxidase blocking (Leica Biosystems). Slides were incubated with primary antibodies followed by application of polymeric HRP-conjugated secondary antibodies (Leica Biosystems). An appropriate Opal Fluorophore-conjugated Tyramide Signal Amplification (TSA) (Akoya Biosciences) was then added at a 1:100 dilution. Slides were rinsed with washing buffer after each step. Following TSA deposition, the slides were again subjected to HIER to strip the tissue-bound primary/secondary antibody complexes and prepare for labeling of the next marker. These steps were repeated until all 6 markers were labeled and finally added with spectral DAPI (Akoya Biosciences) at a 1:10 dilution. Slides were mounted with ProLong Diamond Anti-fade Mountant (Molecular Probes, Life Technologies, Thermo Fisher Scientific) and cured in the dark at room temperature for 24 hours. Ten images (viable tumor regions were selected randomly by pathologists) were acquired for each case using a Vectra 3 Pathology Imaging System Microscope (Akoya Bioscience) and then analyzed and scored by a pathologist using INFORM Software, version 2.4.2 (Akoya Biosciences) (60, 70, 71 ) as well as HALO (Indica Labs) $(26,72)$. The number of immune cells scored was normalized to the number of $\mathrm{ERG}^{+}$(tumor) cells and then $\log _{2}$ transformed before correlating with NanoString data.

Quantification of cell viability. Cell viability was quantified using the CellTiter-Glo 2.0 Luminescent Cell Viability Assay (Promega) according to the manufacturer's protocol. Briefly, cells in exponential growth were harvested by Trypsin-EDTA and seeded at 2000 cells/100 $\mu \mathrm{L} /$ well in 96-well plates and allowed to attach overnight. Various concentrations of drugs were added to cultures in parallel. Control cells were cultured using the same conditions. All reactions were performed in triplicate. Following treatment for 120 hours, the cells were incubated with CellTiter-Glo 2.0 Reagent before absorbances were measured using a 96-well plate reader (M200 Infinite, TECAN). For each treatment, cell viability was evaluated as the percentage of control absorbance. The growth-inhibitory effects were analyzed by generating dose response curves as a plot of the percentage of surviving cells versus the drug concentration. Alisertib, toza- 
A

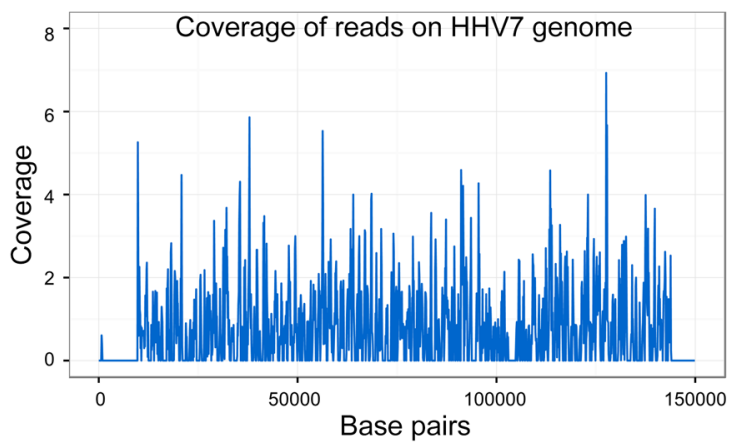

C
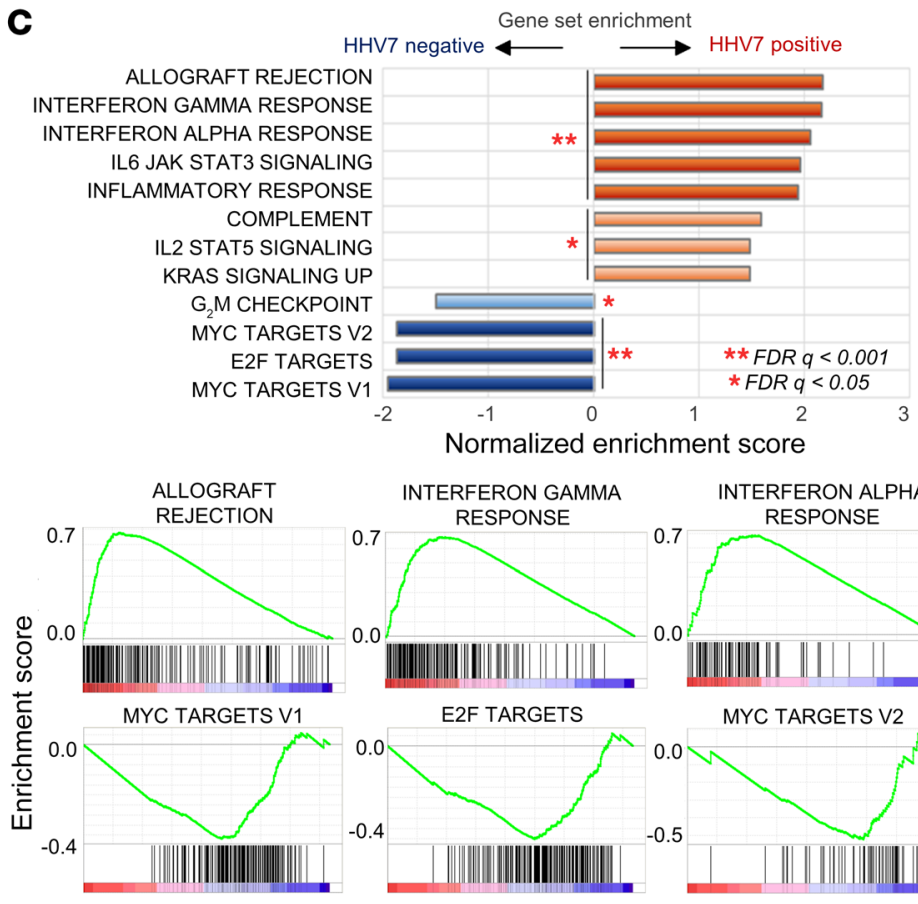

INTERFERON GAMMA INTERFERON ALPHA



0.7 RESPONSE
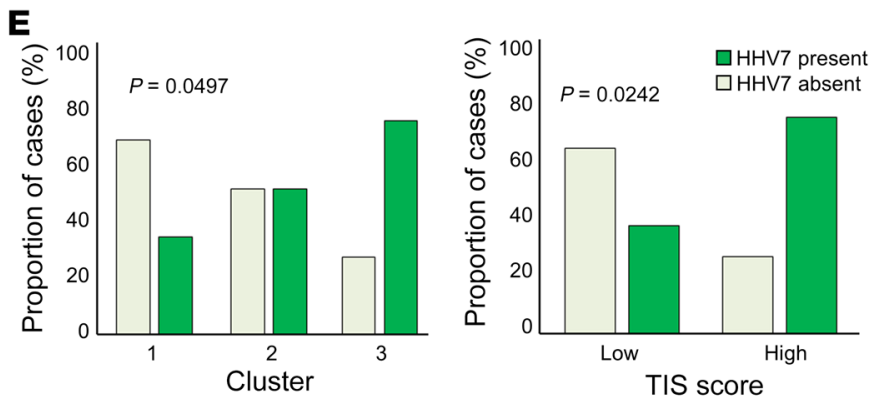

B
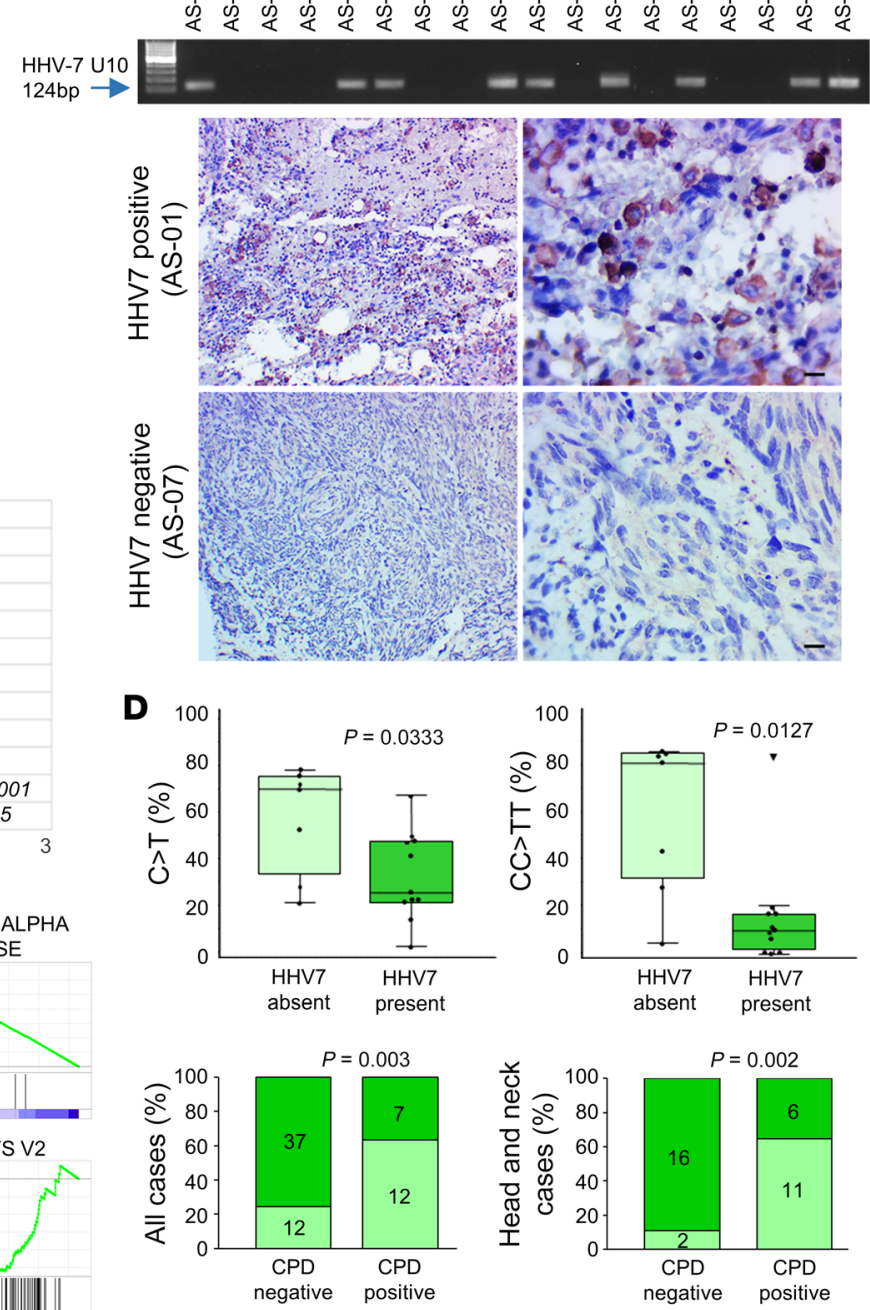

口 HHV7 present $\square$ HHV7 absent

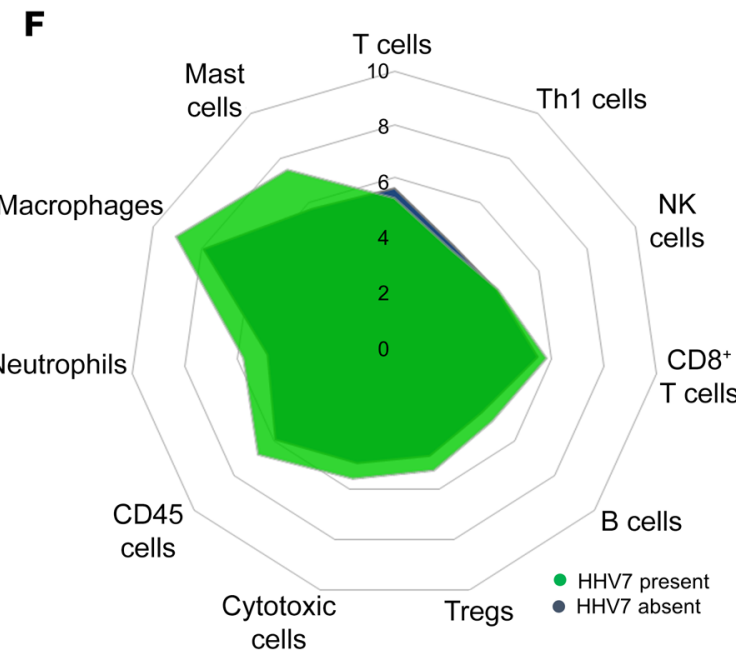

Figure 6. Metagenomic identification of HHV-7 in human angiosarcoma. (A) Viral reads mapped to the HHV-7 genome were detected in the metagenome of scalp angiosarcoma AS-01. (B) The presence of HHV-7 was verified by PCR and/or IHC (scale bars: $20 \mu \mathrm{m}$ ) in 11 of 18 patients (61.1\%) in the discovery cohort.

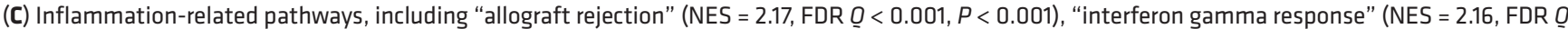
$<0.001, P<0.001$ ), and "interferon alpha response" (NES $=2.06$, FDR $Q<0.001, P<0.001$ ) were significantly enriched in HHV- $7^{+}$angiosarcomas. Virus-negative tumors were enriched for cell cycle-related pathways, including "Myc targets v1" (NES $=-1.95$, FDR $Q<0.001, P<0.001$ ), "E2F targets" (NES $=-1.87$, FDR $O<0.001, P<0.001$ ), and "Myc targets V2" (NES $=-1.87$, FDR $O<0.001, P<0.001$ ). (D) HHV-7+ angiosarcomas had lower levels of C $>$ T mononucleotide substitutions (Mann-Whitney $U, P=0.0333$ ) and CC>TT dinucleotide substitutions (Mann-Whitney $U, P=0.0127$ ). (E) Compared with HHV-7 $7^{-}$cases, HHV-7 ${ }^{+}$ angiosarcomas were associated with high TIS scores (66.7\% vs. $\left.29.4 \%, \chi^{2}, P=0.0242\right)$ and were overrepresented in cluster $3\left(\chi^{2}\right.$ for trend, $\left.P=0.0497\right)$. (F) Virus-positive tumors were strongly enriched for the myeloid compartment, with top cell-type scores for mast cells, macrophages, and neutrophils. 
sertib, barasertib, AZD7762, prexasertib, GDC-0575, volasertib, and GSK461361 were purchased from Selleck Chemicals and prepared according to the manufacturer's recommendations.

Data and materials availability. The WGS data were deposited in the European Genome-phenome Archive (EGA) under accession no. EGAD00001005366. The WTS data were deposited in the EGA under accession no. EGAD00001005367.

Statistics. Comparisons of the frequencies of categorical variables were performed using Pearson's $\chi^{2}$ tests or Fisher's exact tests. Correlation analysis between continuous variables were evaluated by Pearson's correlation coefficient $r$. Growth-inhibitory effects of drugs on cell lines are represented by mean values and their SDs. Continuous variables are represented by box-and-whisker plots, and their associations with categorical variables were evaluated by a Mann-Whitney $U$ or Kruskal-Wallis test as appropriate, or by a Jonckheere-Terpstra test if there was a priori ordering of independent variables. Disease-specific survival was determined by the interval between the date of diagnosis to the date of death from angiosarcoma. Survival was censored at the date of the last follow-up for survivors. Kaplan-Meier analyses were conducted to identify statistically significant univariable predictors of survival, and the results are represented by HRs and 95\% CIs. All statistical analyses were conducted assuming a 2-sided test with a significance level of 0.05 unless otherwise stated and were performed using MedCalc for Windows, version 18.2.1 (MedCalc Software).

Study approval. Written informed consent for the use of biospecimens and clinical data was obtained in accordance with the Declaration of Helsinki. This study was approved by the IRB of the National Cancer Centre Singapore (2010/426/B).

\section{Author contributions}

JYC generated the experimental data and drafted the manuscript. JQL performed the bioinformatics analyses, with support from PG, AB, and KWKS. JY performed multiplex IHC. TKYT and SS provided pathological assessment of tissues. NDMN and JHL were involved in sample preparation. VR, MF, RHHQ, NCT, MCCT and
KCS provided patient samples and clinical data. MM provided cell lines. CKO, DH, JT, ZL, CCYN, and TTT provided technical expertise for various experiments. JYC, BTT, and KCS conceived the study, interpreted the results, and revised the manuscript. SGR, PT, AF, BTT, and KCS directed the study and supervised the research, and all authors read and approved the final version of the manuscript. The order of the co-first authors was assigned on the basis of their relative contributions to the study.

\section{Acknowledgments}

We would like to thank Wei Lin Goh, Jiancheng Hong, Liang Kai Koh, Wei Liu, and Jing Yi Lee, as well as staff members at the SingHealth Tissue Repository and SingHealth Advanced Molecular Pathology Laboratory for technical and administrative assistance. We also thank all of the patients who participated in this study. This work was supported by the Singapore Ministry of Health's National Medical Research Council (NMRC) under a Singapore Translational Research Investigator Award (NMRC/ STAR/0006/2009) and a Research Training Fellowship (NMRC/ Fellowship/0054/2017), as well as by the SingHealth Duke-NUS Academic Medical Centre and the Oncology ACP Nurturing Clinician Scientist Scheme (08-FY2017/P1/14-A28) and a SHF-Foundation Research Grant (SHF/FG653P/2017).

Address correspondence to: Jason Yongsheng Chan, Division of Medical Oncology, National Cancer Centre Singapore, 11 Hospital Drive, Singapore 169610. Phone: 65.6436.8000; Email: jason. chan.y.s@nccs.com.sg. Or to: Bin Tean Teh, Laboratory of Cancer Epigenome, National Cancer Centre Singapore, The Academia, Discovery Tower, Level 8, Singapore General Hospital, 20 College Road, S169865. Phone: 65.6601.1324; Email: teh.bin.tean@ singhealth.com.sg. Or to: Khee Chee Soo, Division of Surgical Oncology, National Cancer Centre Singapore, 11 Hospital Drive, Singapore 169610. Phone: 65.6436.8000; Email: soo.khee.chee@ singhealth.com.sg.
1. Fayette J, et al. Angiosarcomas, a heterogeneous group of sarcomas with specific behavior depending on primary site: a retrospective study of 161 cases. Ann Oncol. 2007;18(12):2030-2036.

2. Young RJ, Brown NJ, Reed MW, Hughes D, Woll PJ. Angiosarcoma. Lancet Oncol. 2010;11(10):983-991.

3. Farid M, et al. Cutaneous versus non-cutaneous angiosarcoma: clinicopathologic features and treatment outcomes in 60 patients at a single Asian cancer centre. Oncology. 2013;85(3):182-190.

4. Guo T, et al. Consistent MYC and FLT4 gene amplification in radiation-induced angiosarcoma but not in other radiation-associated atypical vascular lesions. Genes Chromosomes Cancer. 2011;50(1):25-33.

5. Behjati S, et al. Recurrent PTPRB and PLCG1 mutations in angiosarcoma. Nat Genet. 2014;46(4):376-379.

6. Huang SC, et al. Recurrent CIC gene abnormalities in angiosarcomas: a molecular study of 120 cases with concurrent investigation of PLCG1, KDR, MYC, and FLT4 gene alterations. Am J Surg Pathol. 2016;40(5):645-655.
7. Manner J, et al. MYC high level gene amplification is a distinctive feature of angiosarcomas after irradiation or chronic lymphedema. Am J Pathol.2010;176(1):34-39.

8. Antonescu CR, et al. KDR activating mutations in human angiosarcomas are sensitive to specific kinase inhibitors. Cancer Res. 2009;69(18):7175-7179.

9. Weidema ME, et al. DNA methylation profiling identifies distinct clusters in angiosarcomas. Clin Cancer Res. 2020;26(1):93-100.

10. Painter CA, et al. The Angiosarcoma Project: enabling genomic and clinical discoveries in a rare cancer through patient-partnered research. Nat Med. 2020;26(2):181-187.

11. Danaher P, et al. Pan-cancer adaptive immune resistance as defined by the tumor inflammation signature (TIS): results from The Cancer Genome Atlas (TCGA). JImmunother Cancer. 2018;6(1):63.

12. Kunze K, et al. A recurrent activating PLCG1 mutation in cardiac angiosarcomas increases apoptosis resistance and invasiveness of endothelial cells. Cancer Res. 2014;74(21):6173-6183.
13. Prenen H, et al. Phospholipase C gamma 1 (PLCG1) R707Q mutation is counterselected under targeted therapy in a patient with hepatic angiosarcoma. Oncotarget. 2015;6(34):36418-36425.

14. Italiano A, et al. Alterations of the p53 and PIK3CA/AKT/mTOR pathways in angiosarcomas: a wpattern distinct from other sarcomas with complex genomics. Cancer. 2012;118(23):5878-5887.

15. Murali R, et al. Targeted massively parallel sequencing of angiosarcomas reveals frequent activation of the mitogen activated protein kinase pathway. Oncotarget. 2015;6(34):36041-36052.

16. Calvete $\mathrm{O}$, et al. A mutation in the POT1 gene is responsible for cardiac angiosarcoma in TP53-negative Li-Fraumeni-like families. Nat Commun. 2015;6:8383.

17. Dickson MA, et al. Phase II study of MLN8237 (alisertib) in advanced/metastatic sarcoma. Ann Oncol. 2016;27(10):1855-1860.

18. Subramaniam D, et al. DNA methyltransferases: a novel target for prevention and therapy. Front Oncol. 2014;4:80.

19. Stathis A, Bertoni F. BET proteins as targets for anticancer treatment. Cancer Discov. 
2018;8(1):24-36

20. Kiesel H, et al. Dramatic and durable efficacy of imatinib in an advanced angiosarcoma without detectable KIT and PDGFRA mutations. Cancer Biol Ther. 2009;8(4):319-321.

21. Styring E, et al. Key roles for MYC, KIT and RET signaling in secondary angiosarcomas. Br JCancer. 2014;111(2):407-412.

22. Ayers M, et al. IFN- $\gamma$-related mRNA profile predicts clinical response to PD-1 blockade. JClin Invest. 2017;127(8):2930-2940.

23. Yarchoan M, Hopkins A, Jaffee EM. Tumor mutational burden and response rate to PD-1 inhibition. N Engl J Med. 2017;377(25):2500-2501.

24. Cristescu R, et al. Pan-tumor genomic biomarkers for PD-1 checkpoint blockade-based immunotherapy. Science. 2018;362(6411):eaar3593.

25. Johnson DB, et al. Quantitative spatial profiling of PD-1/PD-L1 interaction and HLA-DR/IDO-1 predicts improved outcomes of anti-PD-1 therapies in metastatic melanoma. Clin Cancer Res. 2018;24(21):5250-5260.

26. Giraldo NA, et al. Multidimensional, quantitative assessment of PD-1/PD-L1 expression in patients with Merkel cell carcinoma and association with response to pembrolizumab. J Immunother Cancer. 2018;6(1):99.

27. Mazzaschi G, et al. Low PD-1 expression in cytotoxic $\mathrm{CD}^{+}$tumor-infiltrating lymphocytes confers an immune-privileged tissue microenvironment in NSCLC with a prognostic and predictive value. Clin Cancer Res. 2018;24(2):407-419.

28. Tumeh PC, et al. PD-1 blockade induces responses by inhibiting adaptive immune resistance. Nature. 2014;515(7528):568-571.

29. Gettinger SN, et al. A dormant TIL phenotype defines non-small cell lung carcinomas sensitive to immune checkpoint blockers. Nat Commun. 2018;9(1):3196.

30. Wong PF, et al. Multiplex quantitative analysis of tumor-infiltrating lymphocytes and immunotherapy outcome in metastatic melanoma. Clin Cancer Res. 2019;25(8):2442-2449.

31. Lu S, et al. Comparison of biomarker modalities for predicting response to PD-1/PD-L1 checkpoint blockade: a systematic review and meta-analysis. JAMA Oncol. 2019;5(8):1195-1204.

32. Yeong J, et al. Multiplex immunohistochemistry/ immunofluorescence (mIHC/IF) for PD-L1 testing in triple-negative breast cancer: a translational assay compared with conventional IHC. J Clin Pathol. 2020;73(9):557-562.

33. Parra ER, et al. Validation of multiplex immunofluorescence panels using multispectral microscopy for immune-profiling of formalin-fixed and paraffin-embedded human tumor tissues. Sci Rep. 2017;7(1):13380.

34. Hoyt C, et al. Abstract LB-318: Multi-institutional TSA-amplified multiplexed immunofluorescence reproducibility evaluation (MITRE study): reproducibility assessment of an automated multiplexed immunofluorescence slide staining, imaging, and analysis workflow. Cancer Res. 2019;79(13 Suppl):Abstract nr LB-318.

35. Wong PF, et al. Multiplex quantitative analysis of cancer-associated fibroblasts and immunotherapy outcome in metastatic melanoma. JImmuno- ther Cancer. 2019;7(1):194.

36. Martinez-Morilla S, et al. Quantitative assessment of PD-L1 as an analyte in immunohistochemistry diagnostic assays using a standardized cell line tissue microarray. Lab Invest. 2020;100(1):4-15.

37. Yeong J, et al. Prognostic value of CD $8{ }^{+} \mathrm{PD}-1^{+}$ immune infiltrates and PDCD1 gene expression in triple negative breast cancer. J Immunother Cancer. 2019;7(1):34.

38. Lim JCT, et al. An automated staining protocol for seven-colour immunofluorescence of human tissue sections for diagnostic and prognostic use. Pathology. 2018;50(3):333-341.

39. Drago F, Ranieri E, Malaguti F, Losi E, Rebora A. Human herpesvirus 7 in pityriasis rosea. Lancet. 1997;349(9062):1367-1368.

40. Wyatt LS, Rodriguez WJ, Balachandran N, Frenkel N. Human herpesvirus 7: antigenic properties and prevalence in children and adults. J Virol. 1991;65(11):6260-6265.

41. Mirandola P, et al. Down-regulation of human leukocyte antigen class I and II and beta 2-microglobulin expression in human herpesvirus-7infected cells. J Infect Dis. 2006;193(7):917-926.

42. Hudson AW, Howley PM, Ploegh HL. A human herpesvirus 7 glycoprotein, U21, diverts major histocompatibility complex class I molecules to lysosomes. J Virol. 2001;75(24):12347-12358.

43. Li H, Durbin R. Fast and accurate short read alignment with Burrows-Wheeler transform. Bioinformatics. 2009;25(14):1754-1760.

44. Tarasov A, et al. Sambamba: fast processing of NGS alignment formats. Bioinformatics. 2015;31(12):2032-2034

45. Wang Q, Jia P, Zhao Z. VERSE: a novel approach to detect virus integration in host genomes through reference genome customization. Genome Med. 2015;7(1):2.

46. Grabherr MG, et al. Full-length transcriptome assembly from RNA-seq data without a reference genome. Nat Biotechnol. 2011;29(7):644-652.

47. Kim S, et al. Strelka2: fast and accurate calling of germline and somatic variants. Nat Methods. 2018;15(8):591-594.

48. Chang X, Wang K. wANNOVAR: annotating genetic variants for personal genomes via the web. JMed Genet. 2012;49(7):433-436.

49. Alexandrov LB, et al. The repertoire of mutational signatures in human cancer. Nature. 2020;578(7793):94-101.

50. Boot A, et al. In-depth characterization of the cisplatin mutational signature in human cell lines and in esophageal and liver tumors. Genome Res. 2018;28(5):654-665.

51. Chen X, et al. Manta: rapid detection of structural variants and indels for germline and cancer sequencing applications. Bioinformatics. 2016;32(8):1220-1222.

52. Mermel CH, Schumacher SE, Hill B, Meyerson ML, Beroukhim R, Getz G. GISTIC2.0 facilitates sensitive and confident localization of the targets of focal somatic copy-number alteration in human cancers. Genome Biol. 2011;12(4):R41.

53. Dobin A, et al. STAR: ultrafast universal RNA-seq aligner. Bioinformatics. 2013;29(1):15-21.

54. O'Leary NA, et al. Reference sequence (RefSeq) database at NCBI: current status, taxonomic expansion, and functional annotation. Nucleic Acids Res. 2016;44(D1):D733-745.

55. Li B, Dewey CN. RSEM: accurate transcript quantification from RNA-seq data with or without a reference genome. BMC Bioinformatics. 2011;12:323.

56. Love MI, Huber W, Anders S. Moderated estimation of fold change and dispersion for RNA-seq data with DESeq2. Genome Biol. 2014;15(12):550.

57. Liberzon A, et al. The Molecular Signatures Database (MSigDB) hallmark gene set collection. Cell Syst. 2015;1(6):417-425.

58. Newman AM, et al. Determining cell type abundance and expression from bulk tissues with digital cytometry. Nat Biotechnol. 2019;37(7):773-782.

59. Stack EC, Wang C, Roman KA, Hoyt CC. Multiplexed immunohistochemistry, imaging, and quantitation: a review, with an assessment of tyramide signal amplification, multispectral imaging and multiplex analysis. Methods. 2014;70(1):46-58.

60. Abel EJ, et al. Analysis and validation of tissue biomarkers for renal cell carcinoma using automated high-throughput evaluation of protein expression. Hum Pathol. 2014;45(5):1092-1099.

61. Lovisa S, et al. Epithelial-to-mesenchymal transition induces cell cycle arrest and parenchymal damage in renal fibrosis. Nat Med. 2015;21(9):998-1009.

62. Garnelo M, et al. Interaction between tumourinfiltrating B cells and T cells controls the progression of hepatocellular carcinoma. Gut. 2017;66(2):342-351.

63. Yeong J, et al. Higher densities of Foxp $3^{+}$regulatory $\mathrm{T}$ cells are associated with better prognosis in triple-negative breast cancer. Breast Cancer Res Treat. 2017;163(1):21-35.

64. Esbona K, et al. COX-2 modulates mammary tumor progression in response to collagen density. Breast Cancer Res. 2016;18(1):35.

65. Mlecnik B, et al. The tumor microenvironment and Immunoscore are critical determinants of dissemination to distant metastasis. Sci Transl Med. 2016;8(327):327ra26.

66. Nghiem PT, et al. PD-1 blockade with pembrolizumab in advanced Merkel-cell carcinoma. N Engl JMed. 2016;374(26):2542-2552.

67. Feng Z, et al. Multispectral imaging of T and B cells in murine spleen and tumor. J Immunol. 2016;196(9):3943-3950.

68. Lam JH, et al. Expression of CD38 on macrophages predicts improved prognosis in hepatocellular carcinoma. Front Immunol. 2019;10:2093.

69. Yeong J, et al. High densities of tumor-associated plasma cells predict improved prognosis in triple negative breast cancer. Front Immunol. 2018;9:1209.

70. Fiore $\mathrm{C}$, et al. Utility of multispectral imaging in automated quantitative scoring of immunohistochemistry. J Clin Pathol. 2012;65(6):496-502.

71. Feng $Z$, et al. Multiparametric immune profiling in HPV- oral squamous cell cancer. JCI Insight. 2017;2(14):93652.

72. Mascaux C, et al. Immune evasion before tumour invasion in early lung squamous carcinogenesis. Nature. 2019;571(7766):570-575. 